\title{
ADVANCED AUTOMATION SOLUTIONS FOR STEELMAKING AND ENVIRONMENTAL PLANTS*
}

\author{
Andreas Rohrhofer ${ }^{1}$ \\ Thomas Kurzmann ${ }^{2}$ \\ Franz Hartl ${ }^{3}$ \\ Philipp Aufreiter ${ }^{4}$
}

\begin{abstract}
Reliable high end automation and process optimization systems are main factors for excellent product quality, safe operation and maximized plant performance in the steel industry and its designated environmental plants. Primetals Technologies introduces new automation packages for consequent safe and cost efficient production. Many areas within the steelplant interested to be monitored and measured automatically are not implemented in the automation system due to limited access or technical reasons. A wireless, self-organizing network facilitates wide ranging data transmission in previously inaccessible areas and opens up a wide range of new opportunities. With Fluid Guard a system to detect leakages with highest accuracy and reacts properly for all kind of water cooled circuits is available, improving the safety in steelmaking process. Lance Guard can be utilized for all fully automatic and semi-automatic lance appliances, but is especially suitable for the products of Primetals Sublance Standard/2.0 and LiquiRob applications, since these systems make seamless integration particularly simple. Manual lance appliances may also be tested and diagnosed. Environmental plants have become a main part in steelmaking plants. Highest performance in combination with optimized energy consumption is the main target in Primetals Technologies' innovations in automation optimization packages for dedusting systems. A new approach for preventive maintenance and operator assistance is introduced with the Acoustic Expert System. In combination with the new Ask cloud based system, Primetals enables a 24/7 monitoring and a whole lifetime observation of any equipment within a steelplant. Furthermore it is now enabled to check the condition of the plant at a fingertip at any time.
\end{abstract}

Keywords: Acoustic; Diagnosis; Condition monitoring; Leakage; EAF; Converter; Environmental; Sample taking; Immersion temperature; Safety; Data based services; Lance measuring; Simulation; Preventive maintenance.

Process Engineering, Primetals Technologies Austria GmbH, Linz, Austria. Process Engineering, Primetals Technologies Austria GmbH, Linz, Austria.

Process Engineering, Primetals Technologies Austria GmbH, Linz, Austria.

Process Engineering, Primetals Technologies Austria GmbH, Linz, Austria. 


\section{INTRODUCTION}

Reliable high end automation and process optimization systems are main factors for excellent product quality, safe operation and maximized plant performance in the steel industry and its designated environmental plants. Primetals Technologies introduces new automation packages for consequent safe and cost efficient production.

Many areas within the steelplant interested to be monitored and measured automatically are not implemented in the automation system due to limited access or technical reasons. A wireless, self-organizing network facilitates wide ranging data transmission in previously inaccessible areas and opens up a wide range of new opportunities.

With Fluid Guard a system to detect leakages with highest accuracy and reacts properly for all kind of water cooled circuits is available, improving the safety in steelmaking process.

Lance Guard can be utilized for all fully automatic and semi-automatic lance appliances, but is especially suitable for the products of Primetals Sublance Standard/2.0 and LiquiRob applications, since these systems make seamless integration particularly simple. Manual lance appliances may also be tested and diagnosed.

Environmental plants have become a main part in steelmaking plants. Highest performance in combination with optimized energy consumption is the main target in Primetals Technologies' innovations in automation optimization packages for dedusting systems. A new approach for preventive maintenance and operator assistance is introduced with the Acoustic Expert System. In combination with the new Ask cloud based system, Primetals enables a 24/7 monitoring and a whole lifetime observation of any equipment within a steelplant. Furthermore it is now enabled to check the condition of the plant at a fingertip at any time

\section{NEW DEVELOPMENTS BY PRIMETALS}

\subsection{Process Data Capturing Beyond Wired Networks}

Many areas are more or less inaccessible for installation of new instrumentation or measurement points because cabling is hard to realize or cost intensive. Therefore wireless, self-organizing network facilitates with wide ranging data transmission opens up a wide range of new opportunities. With WirelessHART additional applications can be realized without cabling or installation of new cable trays. WirelessHART is an open wireless communication standard (IEC 62591, EN 62591) based on the foundation of HART Communication Protocol [2].

To ensure a high overall performance all relevant processes in the steel plant have to be monitored, controlled and coordinated. Limited accessibility or technical reasons are often resulting in inaccurate or delayed measurement data.

Wiplant leaves those limitations behind. The modular system allows reliable measuring of values, conditions etc. using a combination of sensors with a secure data transmission based on the established industrial WirelessHART technology even in harsh environments. An interface to automation solutions enables an advanced process control. 


\subsubsection{Wireless weighing system at scrap chutes or ladle transfer cars}

In order to be consistent with the actual trends of customized steel making processes and effective scrap management a proper weighing measurement system for the used amounts of raw materials becomes more and more in the focus of the plant operators.

For this purpose a high integrated circuit for weighing and temperature measurement purposes has developed shown in Figure 1. The hardware is able to handle up to four weighing disks, two thermocouples and one integrated acceleration sensor for inclination compensation or shock detection.

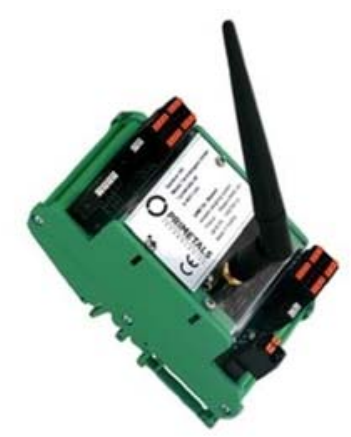

Figure 1: WirelessHART network node for multiple fields of application

In order to use the hardware on transfer cars and scrap chutes with no power supply the main focus of the hardware has been given to a low power realization. Thus lifetimes up to 1 year without recharging the batteries can be achieved. Figure 4 shows the power consumption of the hardware over one year test period. For the pilot test application two batteries with 140 Ah each, parallel connected are used. During the test period the weight of 4 (four) weighing beams and two thermocouples are measured and evaluated. The update rate from WirelessHART network Hardware to the central gateway was set to 4 seconds.

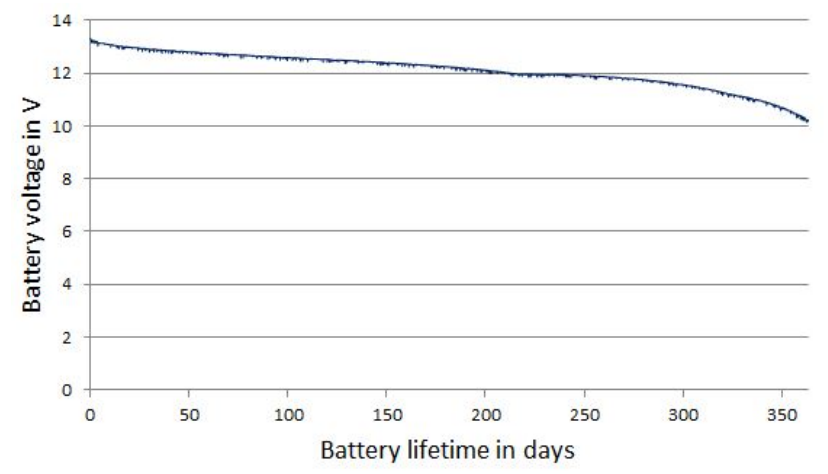

Figure 2: Battery lifetime with WirelessHART data transmission

Typical fields of applications for the wireless weighing systems are continuous ladle weight measurement on transfer cars and slag pots transfer cars or weight measurement on moving scrap chutes.

\subsubsection{Wireless Temperature Measurement at Converter Bottom}

Primetals Technologies recently commissioned a wireless temperature measurement system at a KOBM converter. The configuration consists of 8 thermocouples at the center pipes of the tuyers and one thermocouple for bottom temperature measurement. From the converter bottom the cable route has been laid to the converter shaft (as shown in Figure 3 ) where 3 wireless transmitters have been placed. The temperature readings are transmitted via WirelessHART technology to a gateway located close to the control room. The gateway provides the network device status. 


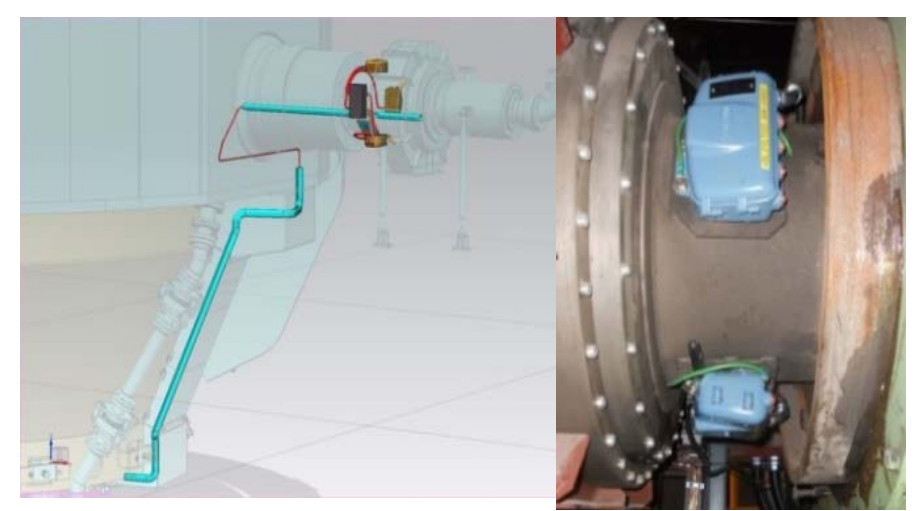

Figure 3: Cable routing and location of wireless transmitters

The reliability of the transmitter increases with the amount of wireless transmitter in the nearby surrounding. For the installed application each transmitter found two or three network participants; the wireless transmitter and the wireless gateway (Figure 4). The reliability of the measurement system was $100 \%$ and there were no interrupts due to closing and opening the doghouse or during the KOBM steel producing process.

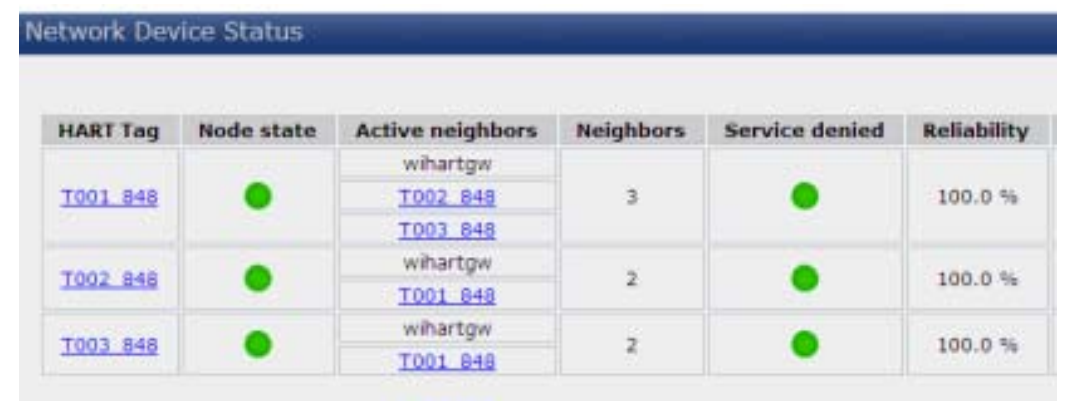

Figure 4: Network device status

\subsubsection{Reliable Vessel Tracking System with wireless data transmission}

The system is based on UHF-RFID (radio-frequency identification) technology. RFID uses radio-frequency electromagnetic fields to transfer data from or to a tag, for the purposes of automatic identification and tracking.

Primetals Technologies developed a new type of passive UHF-RFID Tag called ICETag-RFID developed for the use in harsh environments (shown in Figure 5).

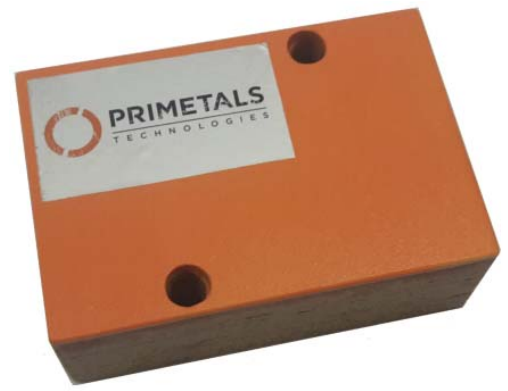

Figure 5: ICE-Tag-RFID; a high temperature UHF-RFID Tag

ICE-Tag-RFID is passive therefore it is powered by the reader - no need of batteries. The Tag has an adequate heat and shock-resistant housing in order to protect it against splashes of liquid metal and mechanical collision.

The operating temperature is up to $200{ }^{\circ} \mathrm{C}\left(392{ }^{\circ} \mathrm{F}\right)$ and it also endures short term temperature peaks up to $800^{\circ} \mathrm{C}\left(1,472{ }^{\circ} \mathrm{F}\right)$. A tag contains electronically stored 
information which can be read within a distance up to $3 \mathrm{~m}$. ICE-Tag-RFID also includes passive damage detection, thus the tracking system recognizes if a tag is damaged and the exchange of the tag can be arranged.

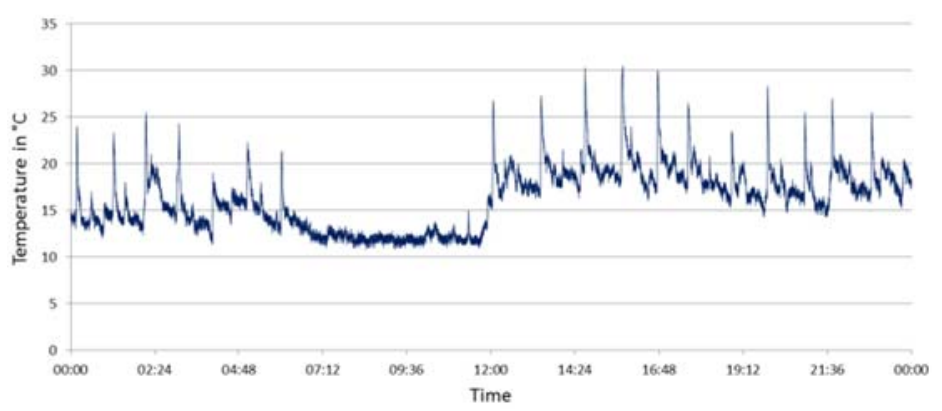

Figure 6: Temperature trend in front of the RFID Antenna

Figure 6 shows the temperature trend in front of a UHF-RFID antenna. Each peak represents a hot vessel passed through the tracking position. Thus a reliable system can provided to detect a vessel beyond the UHF-RFID tracking system. With this temperature evaluation it is possible to detect, if an ICE-Tag-RFID is still mounted on the vessel or it has been broken away e.g. from a crane. In this case the tracking system detects a temperature peak but no ICE-Tag-RFID.

To provide high connectivity a combination of WirelessHART data transmission and the RFID vessel tracking station has successfully tested in a steel plant in Austria.

\subsection{Leakage Detection in Water Cooling Circuits}

Equipment with high temperature exposure in iron and steel making plants is one of the major problems to be solved. The equipment needs adequate protection; most commonly realized method is cooling by water. In case of water leakage within the cooling circuit the reaction of liquid iron or steel can cause injuries to operating personnel, heavy damages to the plant and therefore cost-intensive delays in production. Monitoring of cooling circuits is state of the art and many producers are using industrial instruments and are indicating the process values on the operating system as shown in Figure 7.

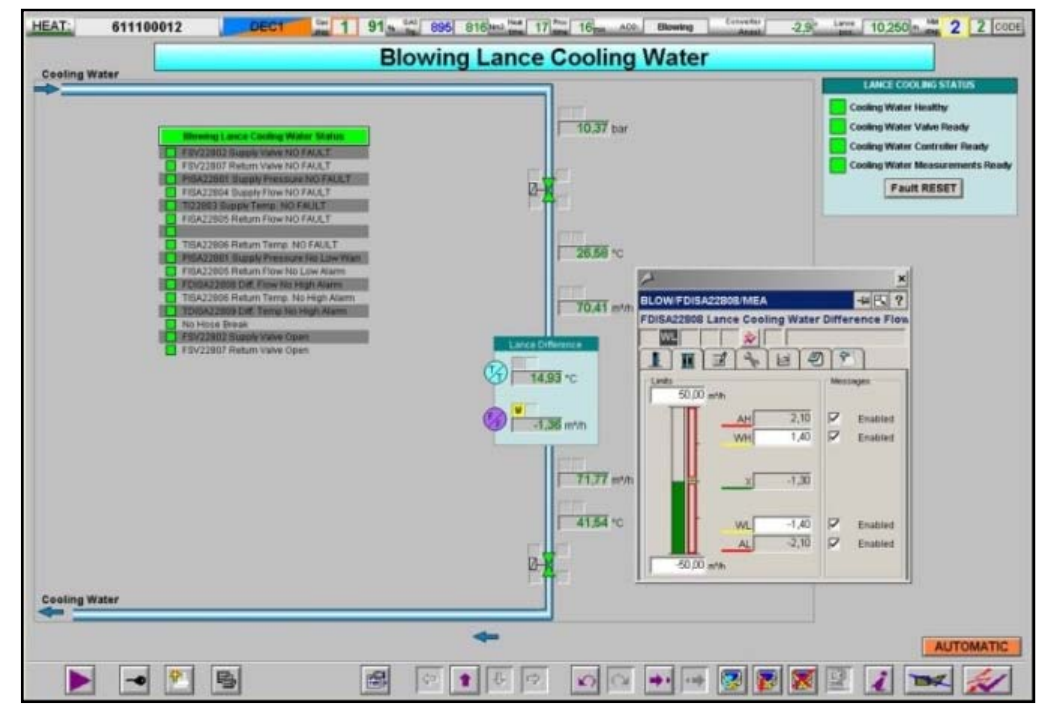

Figure 7: Visualization of cooling water circuit for a top blowing lance 
Fluid Guard is a system with high accuracy for monitoring cooling circuits and detecting water leakages. The system provides fully automatic monitoring and control of the water cooling circuits at highest safety level. The system detects any variation in process conditions and immediate reactions are performed. The safety relevant part of the system is designed in accordance to the standards for "safety of machinery" (EN ISO 13849 series). Furthermore the Fluid Guard system provides an automatic self-diagnostic system where - after predefined time intervals - the system checks itself by detecting self-inflicted virtual water leakages. The measurement of the cooling circuit is mainly realized by use of safety temperature-, pressure and flowmeasurements, safety shut-off valves, self-diagnostic system and a safety control unit as illustrated in Figure 8.

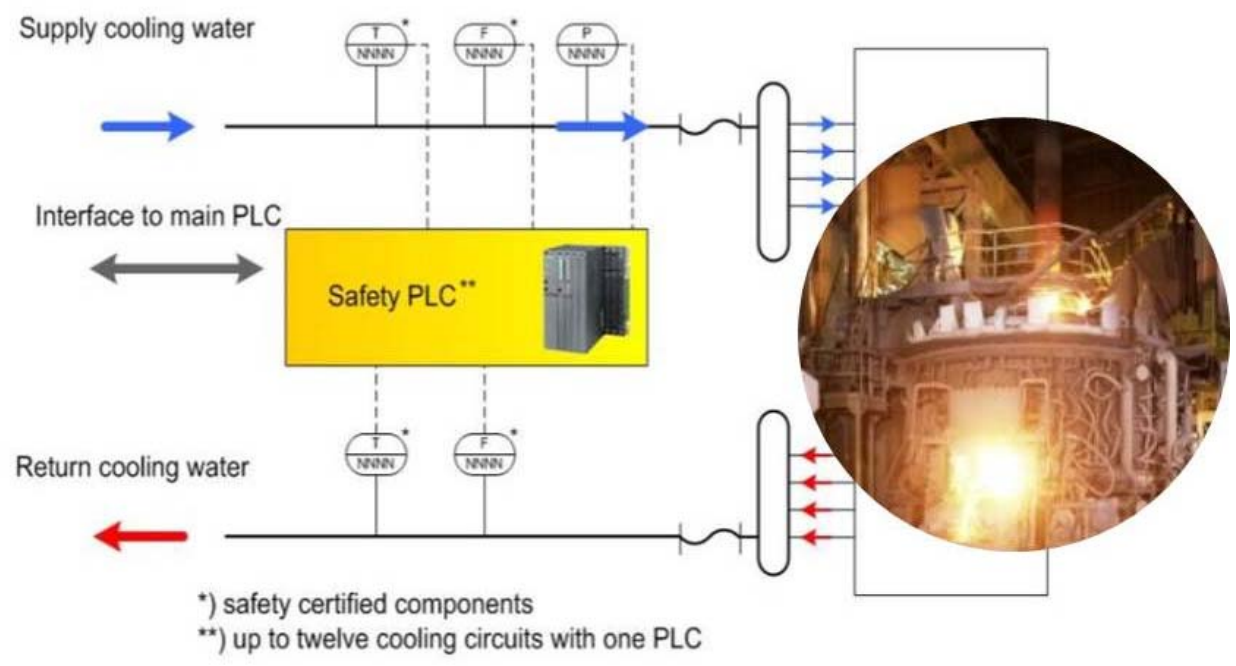

Figure 8: Schematic diagram of Fluid Guard

The software evaluates and compares the measured values from supply and return line and determines the operating flow. Defined counter measures are executed in case of the system is detecting deviations of flow.

The system not only detects leakages; for comprehensive health check the process conditions of the cooling circuits are monitored.

For energy saving Fluid Guard provides a function for dynamic control of the water pumps for cooling circuits. Based on the actual production situation and measurements, the system provides a set-point for drives (VVVF) of the motors for the cooling water pumps which will reduce the energy. According to affinity laws for pumps [2] reduction of rotational speed of $20 \%$ leads to a power reduction of approximately $50 \%$.

\subsection{Lance Guard - Automated Diagnosis System for lance applications}

In typical, lance measuring systems are used to measure steel temperature, liquidus temperature, carbon content and $\mathrm{O} 2$ activity with probes attached to a rod, consisting of a contact block, the probe holder and the lance itself. Measurement is done, by immersion of the probe into the liquid metal. These probes are usually destroyed during a measurement cycle. Remaining a probe too long in the liquid metal often causes damage of the contact block or worse contamination which are unknown and causes great measurement failures (refer to Figure 9). 


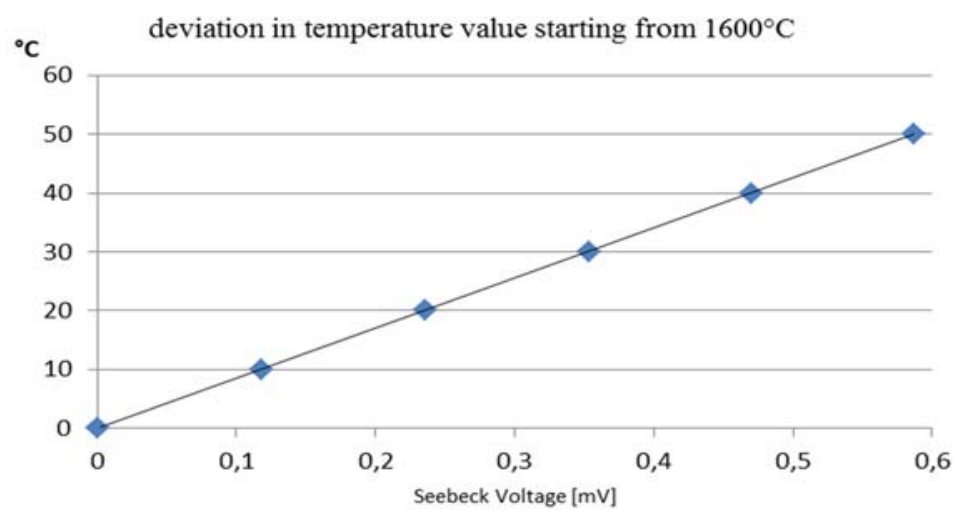

Figure 9: deviation of the measured temperature according to the Seebeck voltage

Figure 9 shows the deviation of the measured temperature according to the Seebeck voltage. Therefore a measurement error at the thermocouple type $S$ of $1.118 \mathrm{mV}$ leads to an $10^{\circ} \mathrm{C}\left(50^{\circ} \mathrm{F}\right)$ Temperature deviation at $1,600{ }^{\circ} \mathrm{C}\left(320^{\circ} \mathrm{F}\right)$ steel bath temperature. Independent from the cold junction temperature which can be compensated, this failure is unknown and therefore it's not possible to compensate it without additional system knowledge and measurement data.

To overcome this problem Primetals developed a fully automatic diagnostics system for continuous testing of the measurement equipment called Lance Guard. The system can be utilized for all fully automatic and semi-automatic lance applications.

Before starting a measurement cycle, a lance adapter has to be applied to the contact block of the measurement lance (Figure 10).

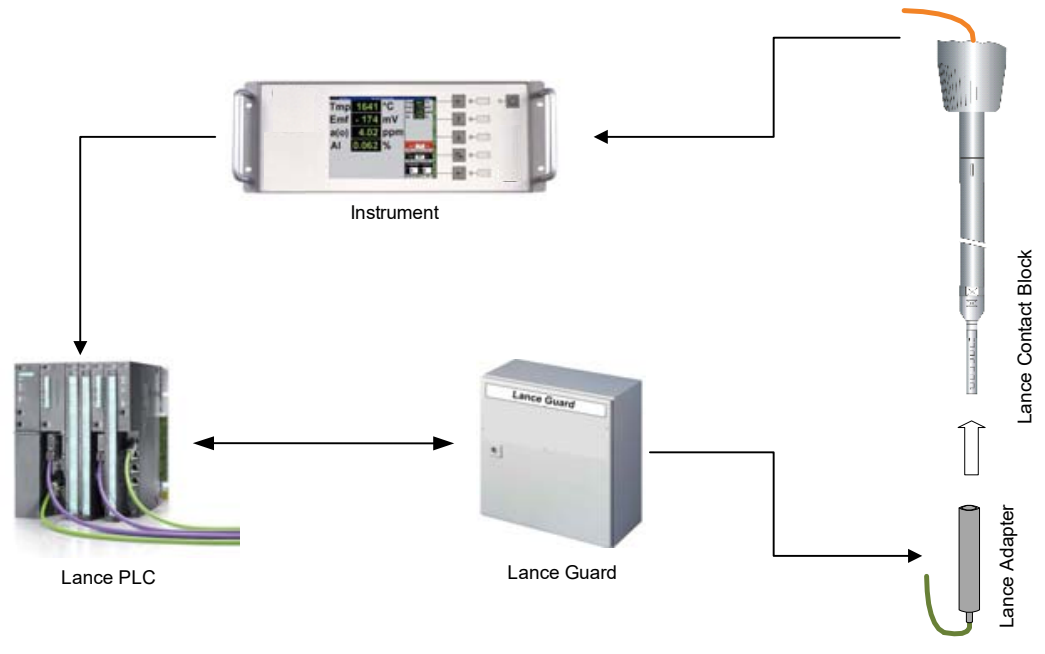

Figure 10: Lance Guard system setup

This could be achieved either buy a robot or a special trained person at a time where the process allows a short interruption. Once the Lance adapter contacted the Contact Block, Lance Guard simulates the whole probe dipping cycle with a high precision voltage output generator. This is done for all channels like temperature, liquidus temperature and $\mathrm{O} 2$ activity. 
Lance Guard temperature value simulation in ${ }^{\circ} \mathrm{C}$

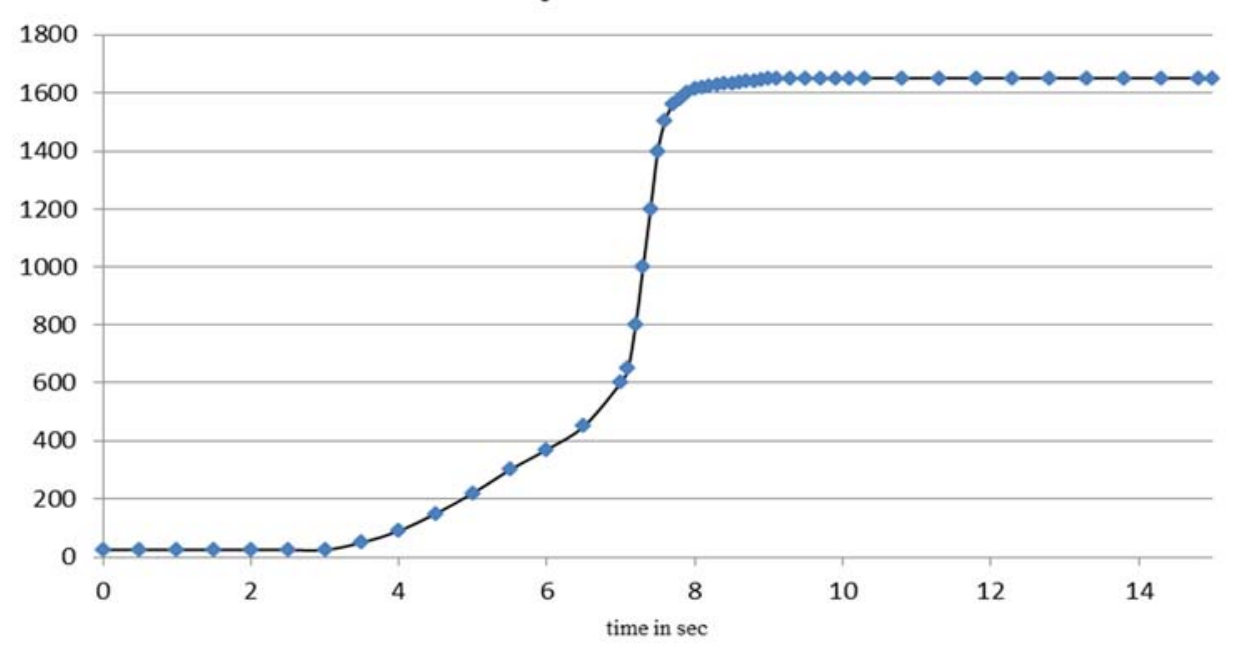

Figure 11: temperature value simulation of a probe dipping cycle

Figure 11 shows the simulated measurement probe temperature trend from a BOF Converter of the Lance Guard system. With respective to real conditions, Lance Guard also simulates the temperature of the slag layer as well as the fast increasing and holding temperature during the dipping process. A Lance Guard simulation cycle without the contacting procedure takes about one minute.

During this simulation cycle, all Lance Guard target values are recorded and compared whit the closed loop feedback from the plant measurement instrument (e.g. Multi-Lab III from Heraeus Electronite). Comparison of the setpoint values from Lance Guard with the actual values from the plant measurement device leads to a detailed quality report.

For example two common detected errors are explained in detail below:

\section{Contamination of the contact block}

One of the main errors in measuring the temperature of the liquid metal phase, utilizing one-way probes is contamination of the contacts at the contact block. According Figure 12, a contamination of a contact causes an additional Seebeck voltage, which can't be compensated, and according to Figure 12 causes dependent from the grade of the contamination an enormous deviation to the real temperature.

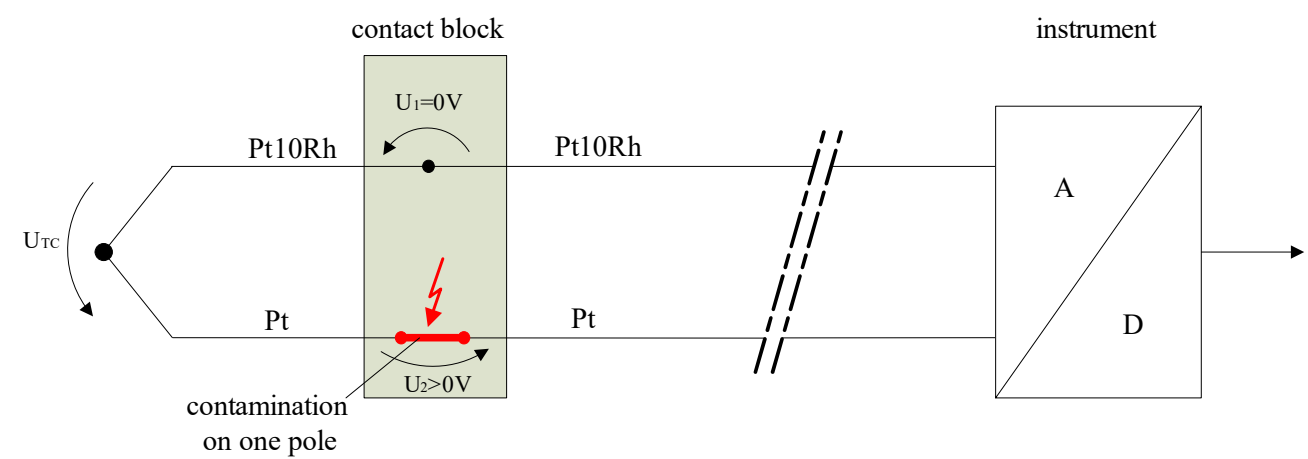

Figure 12: additional Seebeck voltage due to contact contamination 
Figure 12 shows a typical measurement setup with Thermocouple Type-S the two wirings with different materials (Pt10Rh, Pt) and a measurement instrument. Between the instrument and the thermocouple the contact block shows a contamination on one pole. Therefore $U_{1}$ follows to zero because the material doesn't change. Otherwise on the contaminated pole where two additional Seebeck voltages occur as a fact of the material change from Pt to contamination and back.

According to the equation,

$$
U_{T C}+U_{2}=U_{T C, \text { contaminated }}
$$

this additional Seebeck voltages sums up to the thermocouple temperature voltage UTC. Figure 13 shows such a measurement deviation.

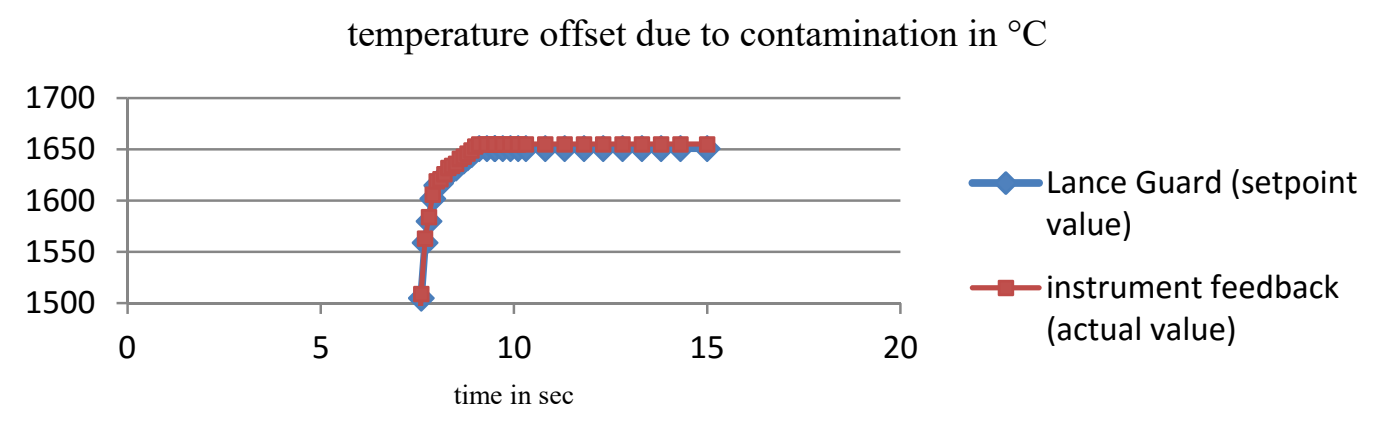

Figure 13: measurement deviation due to contact block contamination

As Figure 13 shows, a permanent offset between setpoint and actual value is detected. This measurement deviation $U_{T C \text {,contaminated is usually in a range between }}$ $1^{\circ} \mathrm{C}\left(34^{\circ} \mathrm{F}\right)$ to $6{ }^{\circ} \mathrm{C}\left(43^{\circ} \mathrm{F}\right)$.

\section{Measurement Deviation caused by cabling}

Another error detectable by the Lance Guard system are random interruptions in the closed loop feedback (Figure 14).

closed loop feedback value interruptions in temperature measurement in ${ }^{\circ} \mathrm{C}$

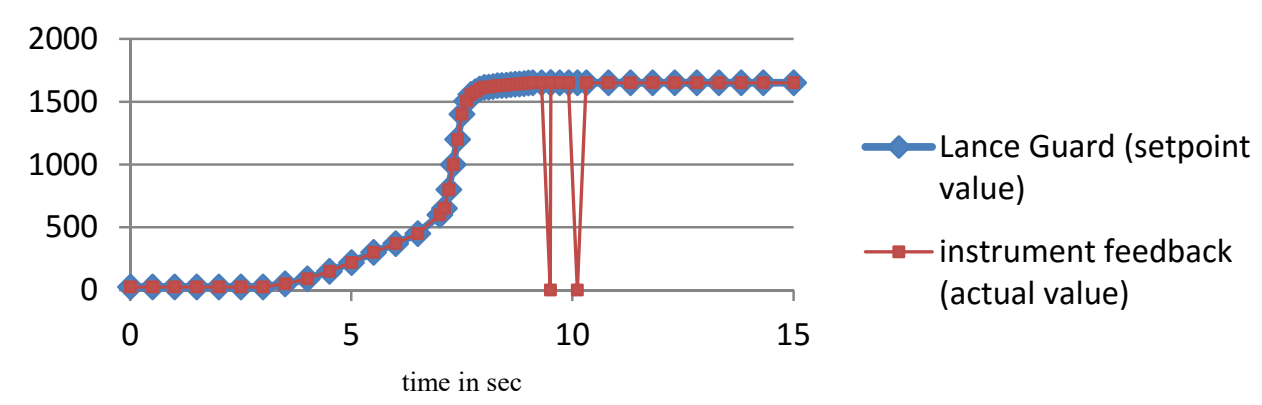

Figure 14: closed loop feedback value interruptions due to damaged wiring

These errors are caused by interferences due to cabling, e.g. cable breakage or ground faults, and appear during movement of the lance only. 


\subsection{Ask - A New Cloud Based Plant Monitoring System}

With Ask, Primetals Technologies introduces a new cloud based monitoring system. Using a cloud the whole monitoring information of a plant could be merged into one single database. Therefore data can be provided in a unique, simple and intuitive way.

The basic idea of the new system is reducing the onsite hardware to a minimum. Thus the ASK hardware replaces comprehensive hardware components, by a simple machine to machine (M2M) communication module with an interface to the customers plant. With this M2M communication only raw data is sent to the cloud, there is no evaluation on site. Thus there is no need for powerful hardware on site, what makes the hardware slim and cheap.

In the cloud system advanced calculations are implemented for a root cause analysis. The results are made available to the end-user with an intuitive app, which can be installed on all kinds of mobile devices like tablets or smartphones.

\section{Acoustic Expert as an implementation example}

One of the first systems implemented to evaluate data in the Ask environment is the Acoustic Expert of Primetals Technologies, a monitoring system which analyses the sound recorded by a microphone to gain information out of it.

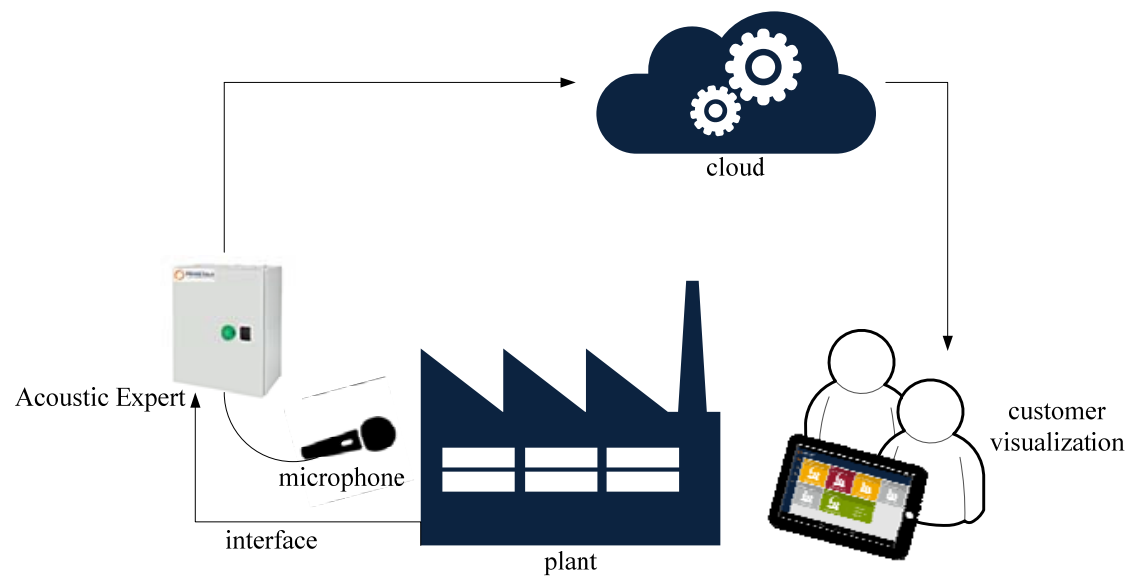

Figure 15: Ask system topology

Figure 15 shows the Ask system topology in combination with the Acoustic Expert. In this Application, the Acoustic Expert System monitors the sound caused by the cleaning valves of a pulse-jet bagfilter plant.

Each sound of a cleaning pulse is recorded in a typically $500 \mathrm{~ms}$ long file and is sent together with a valve identification number to the cloud system where the evaluation and analysis takes place.

The customer is now able to check the condition the pulse-jet bagfilter plant by a fingertip on any time wherever he needs it. 

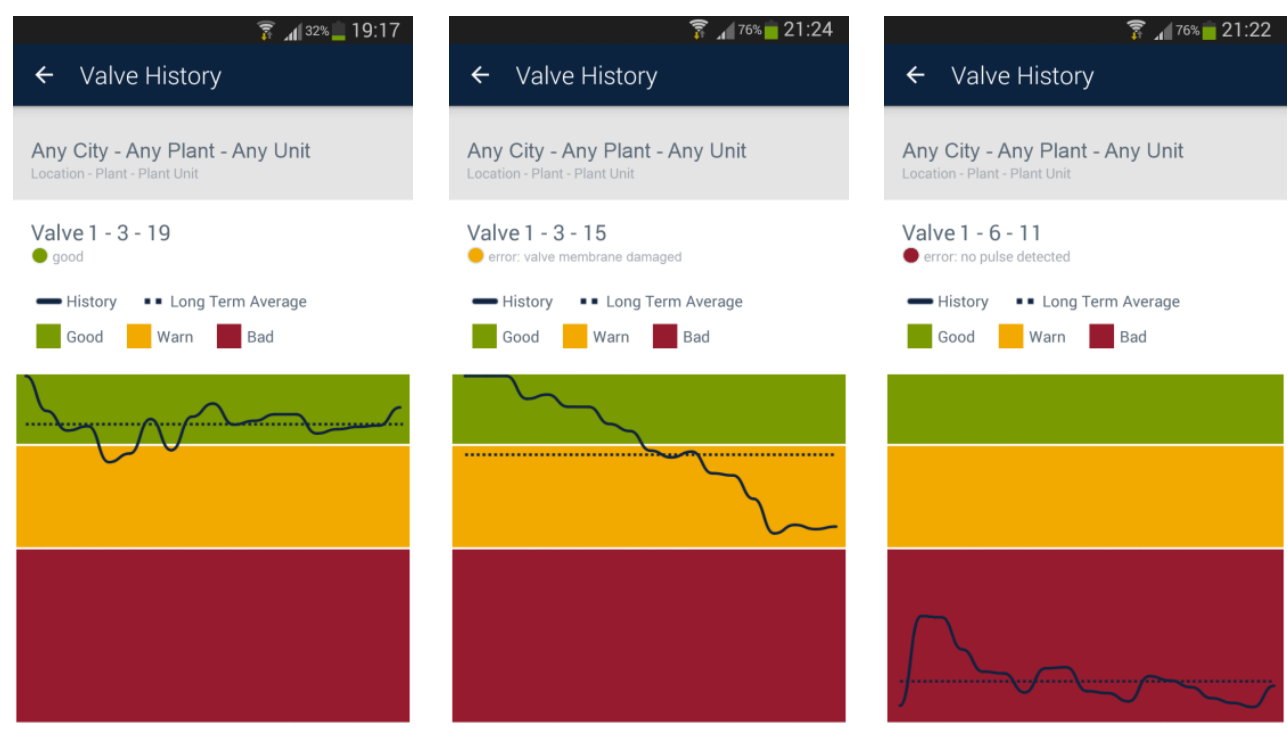

Figure 16: customer visualization

In Figure 16 shows different conditions of three pulse-jet bagfilter valves, monitored with Acoustic Expert and evaluated in the cloud based Ask system. In the usual case, one can see either nearly $100 \%$ or $0 \%$ performance. These states are special cases. In the first case the valve is most of the time performing within the "good"-area, while a decreasing trend in the center picture is shown. A creeping wear of the valve membrane is the conclusion of such a trend. In the last screenshot of the mobile application we can see a valve with approximately $40 \%$ performance.

Figure 16 also shows the way the mobile application provides information. On the one hand there are history information to the observed valve itself, e.g. valve number, the plant and the location of the valve.

\section{CONCLUSION}

With Fluid Guard smallest leakages in water cooling circuits are detected and adequate measures provided, furthermore setpoint for pumps can be calculated and energy savings can be realized.

For retrofitting of weighing equipment on transport cars, Wiplant provide the optimum solution and provide the wireless data transmission for automatic ladle tracking.

Lance Guard is a fully automatic diagnostics system for continuous testing of measurement equipment during the liquid metal phase, utilizing one-way probes.

Lance Guard can be utilized for all fully automatic and semi-automatic lance appliances, but is especially suitable for the products of Primetals Sublance Standard/2.0 and LiquiRob, since these systems make seamless integration particularly simple. Manual lance appliances may also be tested and diagnosed, although in these cases, manual contacting is required.

The Ask cloud based system enables a 24/7 monitoring and a whole lifetime observation of any equipment within a steelplant. Furthermore the customer is now able to check the condition of his plant at a fingertip at any time. 


\section{REFERENCES}

1. T. Kurzmann, F. Hartl, A. Rohrhofer, P. Aufreiter, "Latest Innovations of Technological Automation Packages for Steelmaking Plants" ESTAD 2015 Proceedings, Düsseldorf

2. HART Communication Foundation, WirelesHART Brochure, HCF_LIT-090 Revision 3.1 EN, 2014

3. Pelikan, Bob, The pump book, Fifth Printing, Affinity Law \#3, pg. 116 